\title{
Quantitative experiments with electrons in a positively charged Beam
}

$10 / 27 / 06$
A. W. Molvik, ${ }^{1,2}$ J-L. Vay, ${ }^{1,3}$ M. Kireeff Covo, ${ }^{1,2}$ R. Cohen, ${ }^{1,2}$ D. Baca, ${ }^{1,3}$ F. Bieniosek, ${ }^{1,3}$ A. Friedman, ${ }^{1,2}$ C. Leister, ${ }^{1,3}$ S. M. Lund, ${ }^{1,2}$ P. Seidl, ${ }^{1,3}$ and W. Sharp $^{1,2}$
${ }^{1}$ Heavy-Ion Fusion Science Virtual National Laboratory, Berkeley, CA 94720, USA
${ }^{2}$ Lawrence Livermore National Laboratory, Livermore, CA 94550, USA
${ }^{3}$ Lawrence Berkeley National Laboratory, Berkeley, CA 94720, USA

\begin{abstract}
Intense ion beams are an extreme example of, and difficult to maintain as, a non-neutral plasma. Experiments and simulations are used to study the complex interactions between beam ions and (unwanted) electrons. Such "electron clouds" limit the performance of many accelerators. To characterize electron clouds, a number of parameters are measured including: total and local electron production and loss for each of three major sources, beam potential versus time, electron line-charge density, and gas pressure within the beam. Electron control methods include surface treatments to reduce electron and gas emission, and techniques to remove, or block, electrons from the beam. Detailed, selfconsistent simulations include beam-transport fields, and electron and gas generation and transport, to compute unexpectedly rich behavior, much of which is confirmed experimentally. For example, in a quadrupole magnetic field, ion and dense electron plasmas interact to produce multi-kV oscillations in the electron plasma and distortions of the beam velocity space distribution, without becoming homogenous or locally neutral. [159 words, $\sim 150$ allowed]
\end{abstract}




\section{INTRODUCTION}

Nonneutral plasmas exhibit a wide range of phenomena [1]. We study a configuration consisting of a positive ion beam that has a high rigidity, and to which we add electrons, either intentionally or inadvertently. The ion beam creates a positive space-charge potential of $2 \mathrm{kV}$, which can provide radial confinement of electrons. Electrodes can be biased negatively at either end of a $2 \mathrm{~m}$ long section that includes four quadrupole magnets to transport the ion beam. This provides axial confinement of electrons. The configuration is analogous to an Electron-Beam Ion Trap (EBIT) [2]; it could be termed an Ion-Beam Electron Trap (IBET).

Our work is motivated by the observation that electron clouds and gas pressure rise limit the performance of many major accelerator rings, and may limit linacs being developed as drivers for heavy-ion-inertial fusion (HIF) [3] and for warm dense matter physics (WDM) [4]. We are working to understand the underlying physics and find mitigation mechanisms, through the coordinated application of experiment, theory, and simulation as will be discussed in this paper. Some of our simulation work has been described previously [5]; we will discuss experimental work and more recent simulation results here.

We expect that a major source of electrons and gas in HIF will be loss, or scrape off, of halo ions to the beam tube, which must be as close as possible to the beam envelope in order to reduce the cost of a power plant accelerator. Most of the beam current falls within the beam "envelope", but a small fraction of beam ions undergo radial excursions of the order of a few envelope radii; these are known as halo ions, and are the most likely to be lost by scraping off on the beam tube.

Electron cloud effects typically occur gradually, over many passes of a beam through an accelerator ring. However, we have demonstrated in both experiment and simulation that high electron densities, approaching the beam density, can significantly degrade beam properties in the short distance of four quadrupole magnets (two lattice periods) in a linac [5]. These extreme electron densities produce exaggerated effects on ions, enabling us to validate simulations using a relatively small accelerator. 
Although electrons are generally harmful in accelerators and storage rings, they can be beneficial in particular situations. In particular, longitudinal drift compression of intense space-charge dominated beams to a short length, or focusing to a small radius spot, are usually limited by the beam space charge. Our program has demonstrated that the space charge limits can be removed by performing drift compression and focusing within a plasma that exceeds the compressed and focused beam density and neutralizes the beam space charge. Neutralized drift compression of a $300 \mathrm{kV} \mathrm{K}+$ beam places a head-to-tail velocity tilt on a beam. The tail of the beam then catches up with the head, achieving a factor of 60 compression to a duration of $3 \mathrm{~ns}$ [6]. Neutralization of a beam after the focusing magnets, but not within them, focused a beam to a radius of $0.11 \mathrm{~cm}$, compared with a radius of $1.4 \mathrm{~cm}$ with minimal neutralization [7]. These new capabilities will enable near term WDM experiments using ion beams to heat targets to $\sim 1 \mathrm{eV}$ temperatures [4]. Such benefits of electrons in beams will not be discussed further in this paper.

\section{TOOLS}

Our goal in electron-cloud studies is to use simulations and the HCX experiment to understand electron sources and accumulation in positive charged particle beams, determine effects on the beam, and find mitigation mechanisms. The experimental and simulation tools to accomplish this are discussed in this section.

\section{A. Experimental tools}

The main experimental tool is the High-Current Experiment (HCX), located at Lawrence Berkeley National Laboratory (LBNL). The HCX consists of an injector producing a singly-charged potassium ion beam $(\mathrm{K}+)$ at $1 \mathrm{MeV}$ kinetic energy, followed by a transport lattice made up of an electrostatic quadrupole matching section, a tenquadrupole electrostatic section and a four-quadrupole magnetic section, shown in Fig. 1. The nearly flat top of the beam pulse averages $180 \mathrm{~mA}$ and its duration is $4 \mu \mathrm{s}$, as shown in Fig. 2. Details of the beam transport through 10 electrostatic quadrupoles, preceding the 4 magnetic quadrupoles, have been reported [8]. 
The HCX beam is space-charge dominated in the radial direction, as well as in the axial direction, by the beam space-charge potential of $2 \mathrm{kV}$. The defocusing forces of the beam space-charge, on average, cancel a large fraction of the applied focusing forces of magnetic quadrupoles in the lattice. This results in a factor of 10 reduction in the phase advance of ion oscillations in the presence of space charge relative to a single ion moving in the applied focusing field. The beam potential approaches that of high-energy physics (HEP) and other accelerator rings and is sufficient for many experiments on the generation, accumulation, effects, and mitigation of electron-cloud effects.

The HCX in the region of four magnetic quadrupoles is shown in Fig. 1. To the left is the D2 diagnostic region, an $18 \mathrm{~cm}$ long drift region, between 10 electrostatic quadrupoles and the 4 magnetic quadrupoles. In D2 we measure the beam current and ion phase space distribution, to characterize the beam entering the quadrupole magnets. Similar diagnostics are used after the magnets to determine any changes due to the magnets or to electron clouds. Each magnetic quadrupole has $30 \mathrm{~cm}$ long magnetic field coils in a $47 \mathrm{~cm}$ length elliptical tube that has major and minor inner radii of $5 \mathrm{~cm}$ and 3 $\mathrm{cm}$ respectively. Between each pair of magnets, and after the last one, diagnostic access is provided in a $5 \mathrm{~cm}$ gap, each with 7 ports.

A variety of diagnostics are mounted on the outside of octagonal tubes that fit within the elliptical-bore of quadrupole-magnet beam tubes, as shown in Fig. 3. A gap of about $0.7 \mathrm{~cm}$ annular space is provided between the octagonal mounting tube for diagnostics and the elliptical magnet bore for the recessed diagnostics and cables. The octagonal tubes present a smooth inner surface to the beam, and allow external assembly of the diagnostic arrays. Two different arrays are placed within the third and fourth quadrupoles, and can be interchanged with other arrays. These diagnostics include electrodes shielded from the beam electric field by grids, recessed capacitive electrodes, and $8 \mathrm{~cm}^{2}$ electrodes that are flush with the diagnostics tube in the fourth quadrupole, and will be discussed further in the next subsection $[9,10,11]$.

Other diagnostics are installed between and at the ends of the magnetic quadrupoles: suppressor electrode(s), clearing electrodes to remove electrons, a retarding field analyzer, and the gas electron source diagnostic (GESD) to measure electron emission 
and ion desorption due to ions striking stainless steel surfaces near grazing incidence. These are discussed further below.

A suppressor ring electrode, surrounding the beam after it exits the last quadrupole magnet, is biased to $-10 \mathrm{kV}$ to prevent ion-induced electron emission off an end wall (which can be a slit plate, that can also be biased or grounded) from reaching the magnets, or it can be left unbiased to allow electrons to be emitted from the end wall and to flow into the magnets, flooding them until the average electron line charge in the final magnet is near the value of the beam line charge (line charge is the charge per unit length). In recent experiments, we replaced the ring electrode with a pair of parallel plates - both of these can be biased negatively to suppress electron emission in a manner similar to the ring suppressor; but they can also be biased to produce an electric dipole field across the beam.

As mentioned in the introduction, when the suppressor electrodes are biased negatively, they also prevent electrons from leaving the beam axially at the exit of the fourth quadrupole magnet. The exit electrode of the electrostatic quadrupoles, preceding the magnetic quadrupoles, is also negative; this prevents electrons from being lost upstream of the quadrupole magnets. These electrodes provide axial confinement and the positive ion beam provides radial confinement of electrons, so that electrons are confined in all directions. Once trapped, we expect electrons to remain trapped until the beam potential decreases at the end of the pulse, unless they are removed earlier by clearing electrodes.

Clearing electrodes were installed in the $5 \mathrm{~cm}$ gaps between quadrupole magnets for the purpose of sweeping electrons from each drift region by applying a positive bias voltage. Each clearing electrode is a ring with an inner diameter of $8 \mathrm{~cm}$ and a minor diameter of $1.3 \mathrm{~cm}$, which places the electrodes about $1 \mathrm{~cm}$ outside of the minor radius of the magnet bore such that beam halo ions do not strike the electrodes.

\section{B. Additional experimental tools and applications}

To interpret the electron emission currents from electrodes that scrape the beam halo, we need to know the electron emission and gas desorption coefficients $\gamma_{\mathrm{e}}$ and $\gamma_{0}$ 
respectively, due to ion bombardment of metal surfaces near grazing incidence. In order to infer the current of ions that scrape off, we need to divide the electron emission current by the electron emission coefficient $\gamma_{\mathrm{e}}$. With this, if we also know the gas desorption coefficient $\gamma_{0}$, from the scrape-off current we can infer the total gas desorption.

We designed and commissioned the Gas-Electron Source Diagnostic (GESD) to measure $\gamma_{\mathrm{e}}$ and $\gamma_{0}$, as described in detail in Ref. 12. The GESD has also proven useful for studying mitigation techniques. It is a nearly closed box that includes a small entrance aperture ( 0.3 by $2.5 \mathrm{~cm}$ for the $1 \mathrm{MeV} \mathrm{K}+$ experiments) to admit $\sim 100 \mu \mathrm{A}$ of beam, a small Faraday cup to measure the beam current, a stainless steel target that can be varied in angle relative to the beam, and an ion gauge to measure the gas pressure rise after a pulse. Electron emission coefficients are determined from the ratio of the target emission to the beam current into the GESD. Gas desorption coefficients are determined from the ratio of the pressure rise (in a known volume) to the number of beam ions in a pulse (from integrating the Faraday cup current). We find that $\gamma_{\mathrm{e}} \sim 10^{2}$ and $\gamma_{0} \sim 10^{4}$ for $1 \mathrm{MeV}$ $\mathrm{K}^{+}$ions incident on stainless steel [12]. The electron emission coefficient is observed to vary with the ion angle of incidence $\theta$ as $\gamma_{\mathrm{e}} \propto 1 / \cos (\theta)$, where $d / \cos (\theta)$ is the ion path length through a thin $\mathrm{d} \approx 2 \mathrm{~nm}$ thick surface layer (where the beam-induced emitted electrons originate). Similar scaling of emission with $\theta$ is observed at higher ion energies by Thieberger [13].

These studies of electron emission and gas desorption have increased our understanding of emission and desorption processes, and have yielded new mitigation techniques, as will be discussed. The value of these results greatly exceeds our original goal of calibrating halo scraping diagnostics.

We have studied the scaling of electron emission with $\mathrm{K}+$ ion energy between 50 and $393 \mathrm{keV}$, due to ions impinging at various angles within $10^{\circ}$ of grazing incidence on stainless steel. Our experimental results and modeling for three representative ion energies are shown in Fig. 4. We found that emission scaled with the electronic component of ion stopping in stainless steel, $\mathrm{dE} / \mathrm{dx}$ [14], as has been found previously at higher energies [15]. However, the emission varied more slowly with the ion angle of incidence than $1 / \cos (\theta)$, unlike measurements with $1 \mathrm{MeV} \mathrm{K}+[12]$ and higher energy ions [13]. Based on a modified Sternglass model [16], we have modeled the dependence 
on ion energy and the ion angle of incidence [14], evaluating a $\mathrm{dE} / \mathrm{dx}$ model with the SRIM code [20]. The modelled electron yield in Fig. 4 shows reasonable agreement with measured electron yield for variations of both the ion energy and angle.

Gas can be desorbed from a wall by the impact of lost halo ions, by electron clouds against the wall, or by synchrotron or UV radiation. Gas can degrade a particle beam, directly through stripping or charge exchange of a beam ion with the gas, and indirectly through generation of electrons from ionization of the gas. With $1 \mathrm{MeV} \mathrm{K}+$ ions, approximately $10^{4}$ molecules are generated for each ion striking a stainless steel surface near grazing incidence [12].

The $1 / \cos (\theta)$ variation of electron emission with the ion angle of incidence [highest near grazing incidence $(\theta=90 \mathrm{deg})]$, suggests a mitigation technique: if we sandblast or beadblast a smooth surface, ions that would have struck near grazing incidence now hit the rims of micro-craters at angles closer to normal. This was found to reduce electron emission by an order of magnitude, and gas desorption (which varies much less with angle) by a factor of $\sim 2$ [12]. However, this technique is restricted to ions of low enough energy that their range is less than the thickness of the micro-crater rims. Much higherenergy grazing-incidence ions can penetrate multiple crater rims, generating electrons and gas at each entrance and exit [13]. An axially-thick, antigrazing ring can be used to stop high energy ions at normal incidence, before they strike a wall at grazing incidence [18].

We use a retarding field analyzer (RFA), derived from the popular Rosenberg-Harkay design that uses inexpensive, commercially produced "window frames" as grid mounts, separated by standard alumina-insulators [19]. Our version adds two grids, one that serves as an electron repeller after the entrance aperture, and one that serves as an ion repeller. We provide gaps between grids of 0.8 to $1 \mathrm{~cm}$ to allow bias voltages of $3 \mathrm{kV}$ [20]. This allows it to measure the energy of either ions or electrons. Previous experiments have used similar analyzers to measure the flux and energy of electrons reaching the wall, yielding a qualitative measurement of electron cloud density [21]. We measure the expelled ion energy distribution, which perhaps counter-intuitively provides a more quantitative measurement of electron cloud density than does measuring electrons directly. This is because we measure low energy ions resulting from beam impact on gas. 
These ions are expelled by the beam potential, providing a measurement of the potential, and of the degree to which it is reduced by the accumulation of electrons. From this we infer the electron line charge normalized to the ion line charge [22].

Electron currents to clearing electrodes provide an independent and corroborating measurement of electron line charge as a function of time. When the electrodes are biased positively, they remove electrons that would otherwise accumulate between the negative electrodes at either end of the quadrupole magnets. Integration of this current yields the time-dependent electron line charge that would accumulate in the case when clearing electrodes are off. To obtain the line charge of electrons when clearing electrodes are on, we divide the clearing electrode current by the electron drift velocity in quadrupole magnets $(\sim 0.6 \mathrm{~m} / \mu \mathrm{s})$ [23]. This assumes that the electrons are generated by ion halo impact near the axial center of magnets where the beam-envelope major radius is the largest, a plausible but not yet proven assumption. The line-charge of electrons from clearing-electrode currents agrees with that inferred from RFA measurements [22].

Simulations show that electrons from different sources have differing radial and azimuthal density profiles, within the beam envelope for ionization of gas and extending beyond the beam for the other sources. Each type of electron source can therefor affect an ion beam differently [23]. This motivates developing diagnostics that can measure each major electron source independently. The major types of sources are ionization of gas, electron emission from beam tubes, and electron emission from an end wall. The latter is measured by the current to the most downstream clearing electrode to be biased positively, usually clearing electrode (c). Emission from beam tubes will be discussed later in this section. Measuring the electron source due to ionization of gas involves an issue that we will discuss next.

Electrodes that are exposed to the beam receive a significant capacitive current at the head and tail of the beam (of order $50 \mu \mathrm{A} / \mathrm{cm}^{2}$ ), while the beam potential is changing between 0 and $+2 \mathrm{kV}$. However, we would like to measure particle currents into the wall that are 1 to 3 orders of magnitude smaller than the capacitive currents. These include the ionization of gas by beam-ion impact, which results in an electron that is trapped and an ion that is expelled by the positive beam potential. This current is about 3 orders of 
magnitude smaller than capacitive signals, occurs throughout a pulse, and can be measured during the current flattop.

Another particle current, which we would like to measure, is that due to electrons that have been trapped and accumulate during the pulse, then detrap during the beam tail when the beam potential is falling to zero. The maximum magnitude and polarity of this integrated electron charge at the end of a pulse is the same for a completely neutralized beam as for the integrated charge from a capacitively coupled beam with no electrons. We need to distinguish whether the current is from detrapped electrons or capacitive coupling, furthermore, we would like to be able to measure detrapped electrons that constitute only about $1 \%$ of the beam charge.

Shielding the electrodes with grids achieves the goal of blocking capacitive pickup, and enabling small currents of ions or electrons to be detected. We measured that capacitive pickup could be reduced by a factor of $\sim 30$ with the use of one or by $\sim 500$ with the use of two electromesh grids separated by $400 \mu \mathrm{m}$, each $\sim 5 \mu \mathrm{m}$ thick nickel with a mesh opening center-to-center of $280 \mu \mathrm{m}$ [11].

Gridded ion collectors (GIC), inside quadrupole magnets, are used to determine local densities of gas within an ion beam, and the rate of electron generation by ionization of gas, from the magnitude of the expelled ion current. We collect expelled ions across a quadrupole magnetic field; the magnetic field suppresses electron emission from the collector electrode. The requirement that expelled ions cross the quadrupole field restricts this technique to beams with relatively high space charge and low beam energy (for low quadrupole field strength), so that the beam potential gradient provides sufficient force to drive ions across the quadrupole magnetic field. (This restriction is eliminated for the RFA, which is in a magnetic field-free drift region.) The GICs provide response times of 0.2 to $1 \mu \mathrm{s}$, the time for the $2 \mathrm{kV}$ beam potential to expel ionized residual gas, with masses ranging from hydrogen to argon. This time is substantially less than the beam FWHM of $5 \mu \mathrm{s}$, and provides the capability of measuring the time dependence of desorbed gas reaching the beam [9]. One conclusion from this diagnostic, is that desorbed gas from the walls does not reach the beam within $5 \mu \mathrm{s}$, because the current does not show an upwards inflection partway through the pulse. 
Gridded-electron collectors (GEC), inside quadrupole magnets, are used to collect electrons that detrap while the beam potential is falling to zero at the end of a pulse. For this, it is essential that the GECs are located at azimuths where quadrupole field lines enter the beam tube. Then, electrons that are detrapped, as the beam potential falls to zero at the end of the pulse, can flow along magnetic field lines from the electron cloud to the collector. This azimuthal location does not allow the magnetic suppression of electrons that is achieved with the GIC, which are located at azimuths where magnetic field lines are tangent to the surface [9], but which would also render impossible the collection of magnetized electrons. Therefore we can bias the collector to suppress electron emission from either the collector or the grids, but not from both; with the consequence that the inferences from the GEC are less quantitative than those from the GIC [11].

\section{Simulation tools}

The simulation tool is based on a merge of the Heavy Ion Fusion accelerator particlein-cell (PIC) code WARP [24] and the high-energy physics electron-cloud code POSINST [25], supplemented by additional modules for gas generation and ionization [26] as well as ion-induced electron emission from the Tech-X package TxPhysics [27]. The package allows for multi-dimensional (2-D or 3-D) modeling of a beam in an accelerator lattice and its interaction with electron clouds generated from photon-induced, ion-induced or electron-induced emission at walls, or from ionization of background and desorbed gas. The generation and tracking of all species (beam particles, ions, electrons, and gas molecules) is performed in a self-consistent manner (the electron, ion and gas distributions can also be prescribed -if needed- for special study or convenience). The code runs in parallel and benefits from adaptive mesh refinement [28], particle timestep sub-cycling [29] and a new "drift-Lorentz" particle mover for tracking charged particles in magnetic fields using large time steps [30,31]. These advanced numerical techniques allow for significant speed-up in computing time (orders of magnitude) relative to bruteforce integration techniques, allowing for self-consistent simulations of electron-cloud effects and beam dynamics, which were out of reach with previously available tools. 
We have established a list of different functional modules, and their interrelationships, that are ultimately needed to reach self-consistency for the modeling of HIF beams with e-cloud and gas, and have summarized it in a block diagram (see Fig. 1 of Ref. [31]). We can imagine this as a "roadmap" of a self-consistent modeling capability of electron clouds effects. The initial block of the roadmap is a self-consistent PIC module that follows the beam through an accelerator lattice with its self-field and images at the wall. Ions from the beam halo that strike the wall can desorb neutrals and electrons that have enough time to reach the beam before the end of the pulse, and interact with it. The time-dependent motion of neutrals and electrons must therefore be tracked. The gas can be ionized by beam ions and electrons, leading to new electrons and ions that must be tracked as well. All these particles can hit the walls and produce more neutrals and electrons. Finally, beam ions can be reflected at the wall, and charge-exchange reactions can occur in the gas. More details on the analysis that led to the establishment of this roadmap can be found in Refs. 5,12,27,30.

We follow the convention of labeling the electrons created by the beam hitting the end wall as "primary", while labeling the electrons created by the primary electrons hitting the vacuum pipe surrounding the magnets as "secondary" (these encompass any subsequent generation of electrons). The primary electrons created by ion impact on the end plate and propagating upstream can enter only two quadrants of the fourth (last) magnet (the upper and lower quadrants, as in Fig. 6 of Ref. 5.), and then drift upstream, because the $\mathbf{E} \times \mathbf{B}$ and $\nabla \mathbf{B}$ drifts are both upstream (anti-parallel to the ion beam) in these

quadrants for electrons in the electric field from the $2 \mathrm{kV}$ beam potential. Electrons in the side quadrants drift downstream, parallel to the ion beam.

\section{OSCILLATIONS OF ELECTRONS}

We discovered an unexpected effect while studying electron clouds in ion beams. Experiments show oscillations in the current to a clearing electrode in a drift region between the last two quadrupole magnets when we do not suppress the copious electron emission from the linac end wall, as shown by the red line in Fig. 5(b). Electron suppression is disabled by grounding the suppressor ring electrode, thus allowing 
electrons to propagate upstream. The three clearing electrodes were biased to $+9 \mathrm{kV}$ to remove electrons from the drift regions between quadrupole magnets. The current measured by clearing electrode (c) is compared with simulation in Fig. 5(b). The simulation and experimental results agree on the magnitude and frequency $(\sim 6 \mathrm{MHz})$ of the observed oscillations. WARP PIC code simulations not only show the clearing electrode oscillations that agree with experiment in both magnitude and frequency, as shown by the black line in Fig. 5. The simulations show that electrons increase in density as they stagnate at a quadrupole magnet. Non-linear structures are launched, growing to electron line charges exceeding the ion beam line charge, as shown in Fig. 6.

The current to clearing electrode (b), shown in Fig. 5(a) is an order of magnitude smaller, and does not show oscillations with large amplitude. From comparing the magnitude with that of clearing electrode (c), with and without the suppressor electrode biased, we conclude that clearing electrode (c) removes essentially all the electrons emitted from the end wall, so that clearing electrodes (a) and (b) each collect only electrons that are generated in the adjacent quadrupole magnets. This conclusion is confirmed by other data.

The simulations reveal that these time-dependent oscillations, recorded by clearing electrode (c), are related to axial bunching of electrons. The effect of this bunching is revealed in the plot of line charge densities in Fig. 6. Bunching first appears at the exit of the final quadrupole magnet (at $4.3 \mathrm{~m}$ in Fig. 6) where electron velocities are reduced from a few meters per microsecond thermal velocity to a $0.6 \mathrm{~m} / \mu \mathrm{s}$ drift velocity through a magnet; this stagnation results in higher electron density there. Subsequently, bunching is observed to start near the center of the fourth magnetic quadrupole, where oscillations of large amplitude and wavelength of approximately $5 \mathrm{~cm}$ are observed in the electron density. The effect is so pronounced that the peak electron line charge density reaches 1.75 times the beam line charge density, as shown in Fig. 6.

The bunching of electrons results in radial drifts, apparently due to $\mathbf{E} \times \mathbf{B}$, in addition to the axial $\mathbf{E} \times \mathbf{B}$ and $\boldsymbol{\nabla} \mathbf{B}$ drifts resulting from the unperturbed beam potential, as shown in Fig. 7 where electron bunches are easily observable from the middle of the quadrupole and upstream. (The beam potential is responsible for most of the electron kinetic energy; therefore $\mathbf{\nabla B}$ drifts, as well as $\mathbf{E} \times \mathbf{B}$ drifts, are proportional to the beam potential.) We 
used the 3-D graphics capability of WARP to provide a variety of visual perspectives that help to clarify what is happening, and to evaluate the processes that are occurring. Figure 8 shows a 3-D perspective of conditions similar to those in Fig. 7. The over-neutralization of the beam space-charge (blue) by these electron bunches is evident in Fig. 9, where islands of negative potential of -250 to $-500 \mathrm{~V}$ are formed at the location of the bunches. Closed potential contours are observed to form and drift upstream (to the left) through the magnet. In Fig. 8, we show the primary electron density contours. Primary electrons are emitted from the end wall when it is bombarded by the $1 \mathrm{MeV} \mathrm{K}+$ beam ions. Although some possible candidate explanations have been eliminated, for example the electron-ion two-stream instability [32], the nature of these oscillations has not been firmly identified.

To experimentally benchmark WARP/POSINST simulations of oscillations in electron charge that grow and propagate upstream against the beam, we temporarily replaced the array of diagnostics mounted on an octagonal tube with an axial array of capacitively-coupled electrodes mounted on a tube that snugly fits in the elliptical magnet bore of the fourth magnetic quadrupole. Observing in the simulations that the oscillations begin near the center of the last magnet and propagate upstream against the beam with a frequency of several $\mathrm{MHz}$ and have a wavelength of $\sim 5 \mathrm{~cm}$, we chose the axial locations of electrodes as shown in Fig. 10. The electrodes are $1 \mathrm{~cm}$ long axially, $4 \mathrm{~cm}$ wide azimuthally, and are separated axially center-to-center by $2.5 \mathrm{~cm}$, half the predicted wavelength. The oscillations were predicted to begin near the center of the fourth magnet, which guided the positioning of the electrodes, with an additional electrode $5 \mathrm{~cm}$ further downstream from the center. The quadrupole magnetic field is nearly constant in magnitude between 0 and $\pm 12 \mathrm{~cm}$ (positions indicated by vertical lines in Fig. 10(b)). The magnetic field at the electrode E-10.5 [where the electrode number refers to the axial position of its center $(\mathrm{cm})]$ is down by $4 \%$, the field at the next electrode E-13 is decreased by $50 \%$.

Data from this array are taken at a rate of 1 gigasample/sec (GS/s) for each electrode, and are shown in Fig. 11(a). The data begin with the right hand electrode E+4.5, and progress upstream from there. Electrode locations can be determined from Fig. 10. The spatial growth of the oscillations is hard to see in Fig. 11(a) (but easier in Fig. 12). In Fig. 11(a) the display autoscales the vertical axis so axes values can differ for each channel; 
and in Figs. 11(a) and 11(b) the first $2 \mu \mathrm{s}$ of each frame should be ignored because the oscillations take a few microseconds, in both the simulation and the experiment, to settle to a dominant frequency and grow to their saturated state. After this, downstream channels are more quiescent, while upstream channels through E-13 grow in amplitude. The four electrodes at the left side (upstream end) of Fig. 10: E-15.5, E-18 and E-20.5 are in the fringe field of the quadrupole magnet, and show progressively smaller signals as the magnetic field decreases.

In Fig. 11(b), we show the simulated data for each channel, with a constant plot scale, so the spatial growth in oscillation amplitude is more readily apparent here as we advance from left to right. Significant similarities are observed with the measured data. The positive signal at the beginning is due to capacitive pickup from the rising beam potential at the head of the beam. Shortly after the head, electron oscillations begin and continue throughout the duration of the pulse. The horizontal scales differ, between Figs. 11(a) and 11(b), because the digital oscilloscopes are triggered by a separate signal that is offset from the beam arrival time.

We compute the oscillation power spectrum by squaring the absolute value of the fast Fourier transform (FFTs) of each channel between 5 and $6 \mu$ s [Fig. 11(a)] and dividing by the number of data points. We plot the results in Fig. 12, averaged over 1 to $31 \mathrm{Mhz}$, as a function of electrode position (from Fig. 10). The spatial growth of the oscillations is clear. The oscillations begin near the center of the last quadrupole magnet and grow in the upstream direction, until the magnetic field magnitude begins to decrease at $15 \mathrm{~cm}$ (E-15.5 and beyond).

In WARP, the spatial growth of oscillations is computed from a Fourier transform as in the experiment, and is also shown in Fig. 12. The measurements agree with simulations, finding the same wavelength $(5 \mathrm{~cm})$, frequency range (5-15 Mhz), and amplitude of oscillations. As the oscillations move upstream, both WARP and the experiment show the oscillation amplitude growing spatially faster than it did near the magnet center. The agreement is very good, except that the simulation shows the amplitude decreasing beyond the end of the constant quadrupole field where the magnetic field has dropped to 0.5 of the central value, whereas the experimental amplitude doesn't decrease until the next electrode at $17.5 \mathrm{~cm}$ (not shown) where the magnetic field is 0.1 
of the central value. The discrepancies may be due to small differences, between the experiment and simulations, in the fall-off of the magnetic field near the ends of the magnet, and to small $(\sim 1 \mathrm{~mm})$ axial errors in locating the electrode array in HCX, but we have not demonstrated this.

Experiments and simulations are continuing to improve our understanding of the mechanism responsible for the oscillations, and the conditions necessary for the oscillations to exist. From simulations where the beam radius is varied, we observe that the further electrons extend outside the ion beam, the greater the oscillation amplitude.

Motivated by these simulations, we have taken data similar to that in Figs. 11, except that we varied the end-wall potential. Biasing the end-wall positively is expected to have two effects: (1) Electrons emitted from the end wall will not extend radially as far outside of the beam, which by analogy with the simulations should reduce the oscillation amplitude. In addition, these electrons should not be able to reach the beam tube at ground potential to generate secondary electrons. (2) The beam potential should no longer be reduced to near zero when flooded with electrons but should be reduced only to a potential near the end-wall potential. We observe that biasing the end wall to $+500 \mathrm{~V}$ reduces the oscillation amplitude to near zero, see Fig. 13. This is in qualitative agreement with the simulations, however quantitative comparison will await subsequent simulations that directly simulate the experimental conditions, including effect (2) above.

In conclusion, we have developed a variety of diagnostics that measure the three major types of sources of electrons - ionization of gas, emission from beam tubes, and emission from an end wall. We have measured the time-dependent absolute magnitude of electron accumulation in an ion beam. We have measured electron emission and gas desorption yields and increased the understanding of the scalings and processes involved. We have observed oscillations of dense electron clouds in both experiment and simulation, experimentally validating the simulations by detailed quantitative agreement with the magnitude and time dependence of measured data from each channel of an 8 channel array. These data validated the simulation predictions of frequency content, wavelength, and the oscillation power and measured spatial growth rates. Further experiments and simulations of varying the relative radii of the electron cloud and the ion beam yielded qualitatively similar effects on the oscillation amplitudes, and here we plan further 
simulations that will more closely match the experimental conditions for a quantitative comparison.

\section{Acknowledgements}

Experimental operations at LBNL were ably supported by Wayne Greenway, Ralph

Hipple, William Strelo, Ron Beggs, Takeshi Katayanagi, Craig Rogers, and Edgardo

Romero II. This work performed under the auspices of the U.S Department of Energy by

University of California, Lawrence Livermore and Lawrence Berkeley National

Laboratories under contracts No. W-7405-Eng-48 and DE-AC02-05CH11231. 


\section{References}

${ }^{1}$ R. C. Davidson, Physics of Nonneutral Plasmas, (Addison Wesley, Redwood City, CA, 1990), p. 1.

${ }^{2}$ R. Becker, Rev. Sci. Instrum. 71, 816 (2000).

${ }^{3}$ B. G. Logan, F. M. Bieniosek, C. M. Celata, et al., "Overview of US heavy ion fusion research,” Nuclear Fusion 45, 131-137 (2005).

${ }^{4}$ N. A. Tahir, C. Deutsch, V. E. Fortov, V. Gryaznov, D. H. H. Hoffmann et al., Phys. Rev. Lett. 95, 035001 (2005); R. More, H. Yoneda and H. Morikami, J. Quant. Spectrosc. \& Radia. Transfer 99, 409 (2006); J. J. Barnard, J. Armijo, R. M. More, et al., "Theory and Simulation of Warm Dense Matter Targets," Submitted to Nucl. Instrum. Methods A.

${ }^{5}$ R. H. Cohen, A. Friedman, M. Kireeff Covo, S. M. Lund, and A. W. Molvik, F. M. Bieniosek, P. A. Seidl, and J.- L. Vay, P. Stoltz, and S. Veitzer, Phys. Plasmas 12, $056708(2005)$.

${ }^{6}$ P. K. Roy, S. S. Yu, E. Henestroza, et al., Phys. Rev. Lett. 95, 234801 (2005).

${ }^{7}$ P. K. Roy, S. S. Yu, S. Eylon, et al., Phys. Plasmas 11, 2890 (2004).

${ }^{8}$ L. R. Prost, P. A. Seidl, F. M. Bieniosek, C. M. Celata, A. Faltens, D. Baca, E. Henestroza, J. W. Kwan, M. Leitner, W. L. Waldron, R. Cohen, A. Friedman, D. Grote, S. M. Lund, A. W. Molvik, and E. Morse, Phys. Rev. ST - Accel. Beams 8, 020101 (2005).

${ }^{9}$ A.W. Molvik, P. A. Seidl, F. M. Bieniosek, R. H. Cohen, M. Kireeff Covo, L. Prost, "Experimental Studies of Electrons in a Heavy-Ion Beam", Nucl. Instrum. Methods A, 544, 194-201 (2005).

${ }^{10}$ F. M. Bieniosek, S. Eylon, A. Faltens, A. Friedman, J. W. Kwan, M. A. Leitner, A. W. Molvik, L. Prost, P. K. Roy, P. A. Seidl, G. Westenskow, Nucl. Instrum. Methods A 544, 268-276 (2005). 
${ }^{11}$ A.W. Molvik, M. Kireeff Covo, R. H. Cohen, A. Friedman, W. M. Sharp, David Baca, F. M. Bieniosek, C. Leister, P. A. Seidl, J-L. Vay, "Quantitative electron and gas cloud experiments," submitted to Nucl. Instrum. Methods A.

${ }^{12}$ A. W. Molvik, M. Kireeff Covo, F. M. Bieniosek, L. Prost, P. A. Seidl, D. Baca, A. Coorey, and A. Sakumi, Phys. Rev. ST Accel. Beams 7, 093202 (2004).

${ }^{13}$ P. Thieberger, A. L. Hanson, D. B. Steski, V. Zajic, S. Y. Zhang, and H. Ludewig, Phys. Rev. A 61, 042901 (2000).

${ }^{14}$ Michel Kireeff Covo, Arthur Molvik, Alex Friedman, Glen Westenskow, John J. Barnard, Ronald Cohen, David Grote, and Steven M. Lund, Peter Seidl, Joe W. Kwan, Grant Logan, David Baca, Frank Bieniosek, Christine M. Celata, and Jean-Luc Vay, Jasmina L. Vujic, Phys. Rev. ST Accel. Beams 9, 063201 (2006).

${ }^{15}$ H. Rothard K. Kroneberger, A. Clouvas, E. Veja, P. Lorenzen, N. Keller, J. Kemmler, W. Meckbach, and K.-O. Groeneveld, Phys. Rev. A 41, 2521 (1990).

${ }^{16}$ E. J. Sternglass, Phys. Rev. 108, 1 (1957).

${ }^{17}$ J. F. Ziegler, http://www.srim.org/.

${ }^{18}$ P. Thieberger, W. Fischer, H. Hseuh, V. Ptitsyn, S. P. Snydstrup, and S. Y. Zhang, Phys. Rev. ST Accel. Beams 7, 093201 (2004).

${ }^{19}$ R. A. Rosenberg and K. Harkay, Nucl. Instrum. Methods A 453, 507 (2000).

${ }^{20}$ M. Kireeff Covo, A. W. Molvik, A. Friedman, J. J Barnard, P. A. Seidl, B. G. Logan, D. Baca, and J. L. Vujic, "Beam Interaction Measurements with a Retarding Field Analyzer in a High-Current High-Vacuum Positively-Charged Particle Accelerator," submitted to Nucl. Instrum. Methods A.

${ }^{21}$ K. Harkay and R. Rosenberg, Phys. Rev. ST Accel. Beams 6, 034402 (2003).

${ }^{22}$ Michel Kireeff Covo, Arthur Molvik, Alex Friedman, Jean-Luc Vay, Peter Seidl, Grant Logan, David Baca, and Jasmina L. Vujic, Phys. Rev. Lett. 97, 054801 (2006).

${ }^{23}$ R. H. Cohen, A. Friedman, S. Lund, A. W. Molvik, ,E. P. Lee, T. Azevedo, J.-L. Vay, P. Stoltz, and S. Veitzer, Phys. Rev. ST Accel. Beams 7, 124201 (2004). 
${ }^{24}$ D. P. Grote, A. Friedman, I. Haber, Fus. Eng. \& Des. 32-33, 193 (1996), available at http://hif.lbl.gov/theory/WARP.summary.html

${ }^{25}$ M. T. F. Pivi and M. A. Furman, Phys. Rev. ST Accelerators and Beams 6, 034201 (2003).

${ }^{26}$ J.-L. Vay, M. A. Furman, P. A. Seidl, LBNL, R. H. Cohen, A. Friedman, D. P. Grote, M. Kireeff Covo, A. W. Molvik, LLNL, P. H. Stoltz, S. Veitzer, Tech-X Corp., J. Verboncoeur, UC Berkeley, "Filling in the Roadmap for self-Consistent Electron Cloud and Gas Modeling," Proceedings of the 2005 Particle Accelerator Conference, Knoxville, TN, May 16-20, 2005.

${ }^{27}$ P. H. Stoltz, M. A. Furman, J.-L. Vay, A. W. Molvik, and R. H. Cohen, Phys. Rev. ST Accel. Beams 6, 054701 (2003); P. Stoltz, S. Veitzer, R. Cohen, A. W. Molvik, and J.L. Vay, Phys. Rev. ST Accel. Beams 7, 103201 (2004).

${ }^{28}$ J.-L Vay, P. Colella, J. W. Kwan, et al, Phys. Plasmas 11, 2928 (2004).

${ }^{29}$ J.-C. Adam, a. Gourdin Serverniere, A. B. Langdon, J. Comput. Phys. 47, 244 (1982).

${ }^{30}$ R. H. Cohen, A. Friedman, D. P. Grote, J.-L. Vay, "Large-timestep mover for particle simulations of arbitrarily magnetized species," Submitted to Nucl. Instrum. Methods A.

${ }^{31}$ J.-L. Vay, M. A. Furman, P. A. Seidl, et al., "Self-consistent simulations of heavy-ion beams interacting with electron-clouds," Submitted to Nucl. Instrum. Methods A.

${ }^{32}$ Qin H, E. A. Startsev, R. C. Davidson, Phys. Rev. ST Accel. Beams 6, 014401 (2003). 
Fig. 1. (Color online) The magnetic quadrupole region of HCX, from the D2 diagnostic region on the left to the D-End diagnostic region beginning on the right is shown in an elevation view. The lattice half-period length is $0.52 \mathrm{~m}$. Clearing electrodes a, b, and c are shown in the drift regions between each pair of quadrupoles. A suppressor electrode prevents beam-induced electron emission, from structures hit by beam in D-End, from reaching the quadrupole magnets. Vacuum pumps are located in each of the diagnostic tanks a-c, as well as at both ends of the region shown. Gas can be fed at tank b. The cylindrical suppressor ring has been replaced with 2 separately biased plates that can perform additional functions.

Fig. 2. Beam current of $1 \mathrm{MeV} \mathrm{K}+$ ions, measured with a Faraday cup.

Fig. 3. Octagonal tube for mounting multiple diagnostics inside an elliptical-bore quadrupole magnet.

Fig. 4. (Color online) Ion-induced electron emission yields, measured and modeled, with aid of TRIM code, for three energies of potassium ions.

Fig. 5. (Color online) Currents to clearing electrodes (b) and (c), showing both measurements (red) and simulations (black).

Fig. 6. (Color online) Line charge of the ion beam, and electrons in the fourth quadrupole magnet, after oscillations in electron cloud are saturated.

Fig. 7. (Color) Warp simulation results in the Y-Z plane for the 4 magnetic quadrupoles, showing the ion beam (black), primary electrons from ions impacting a wall (red), and secondary electrons from electrons impacting a wall (blue). Each color is opaque and hides the other species. Oscillations are seen in the primary electron radii in magnet 4, identified by a circle. 
Fig. 8. (Color online) The charge density of primary electrons (color coded) emitted from the end wall extending from the drift region between the last two magnets on the left, through the last magnet, to the grounded end wall on the right.

Fig. 9. (Color online) The potential contours (color coded) extending from the drift region between the last two magnets on the left, through the last magnet, to the grounded end wall on the right.

Fig. 10. The location of capacitively coupled electrodes in the beam tube of the last quadrupole magnet is indicated, relative to the center of the magnet. (BPM stands for Beam-Position Monitor, a frequent application of capacitively-coupled electrodes. An array of 11 electrodes is distributed axially in the bottom quadrant. At -12.5 to $-13.5 \mathrm{~cm}$, an azimuthal array of electrodes, one electrode per quadrant is provided. Top and bottom quadrant electrodes extend $1 \mathrm{~cm}$ axially and $4 \mathrm{~cm}$ azimuthally. Side quadrant electrodes extend $1 \mathrm{~cm}$ axially and $6 \mathrm{~cm}$ azimuthally.

Fig. 11. Data from 10 channels of the axial array: (a) experiment, (b) simulation.

Fig. 12. The results from Fig. $\mathrm{C}$ are integrated over a frequency range of 4 to $8 \mathrm{MHz}$, and plotted as a function of electrode position (from Fig. 6). the oscillations begin near the center of the last quadrupole magnet and grow in the upstream direction, until the magnetic field magnitude begins to decrease at $15 \mathrm{~cm}$. Experimental measurements (blue triangles), WARP simulation of experiment (tan circles).

Figure 13. (Color online) We compare the measured oscillating line charge to that of the beam $(0.082 \mu \mathrm{C} / \mathrm{m})$, as a function of the end-wall bias voltage. 
Figures

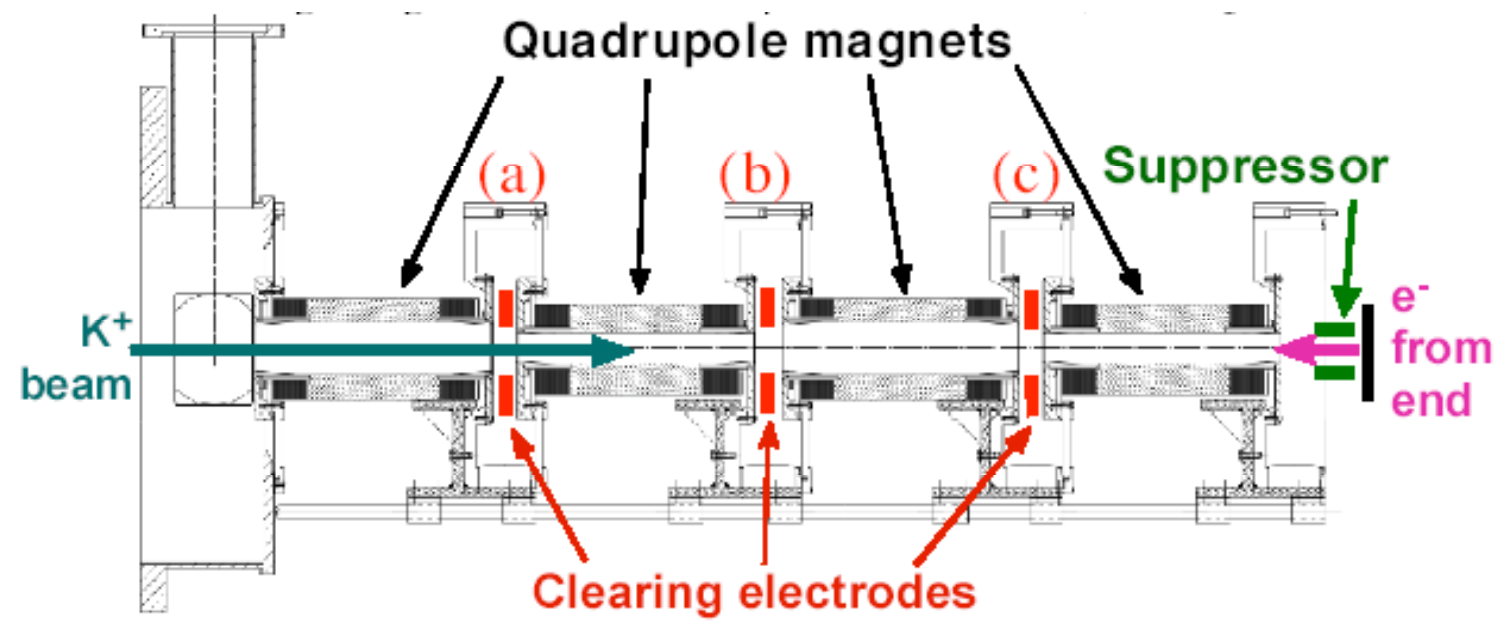

Fig. 1.

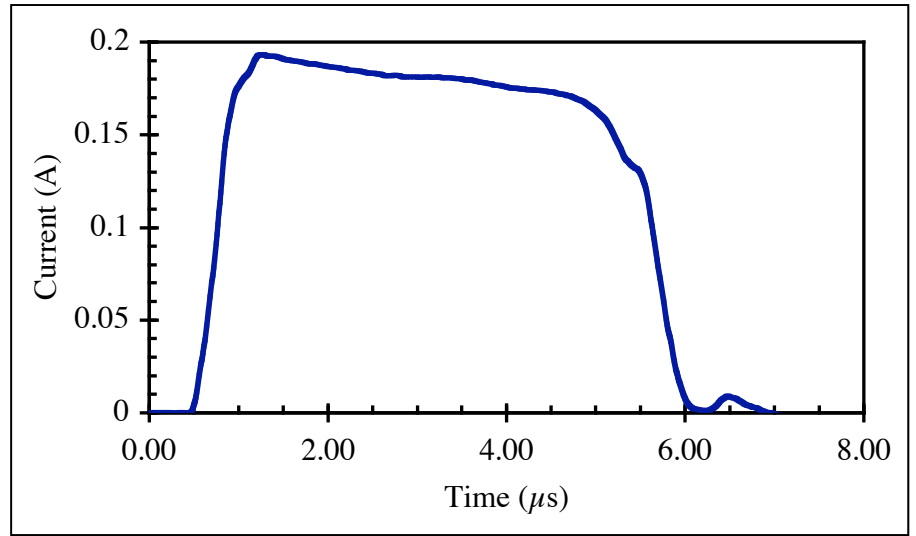

Fig 2. 


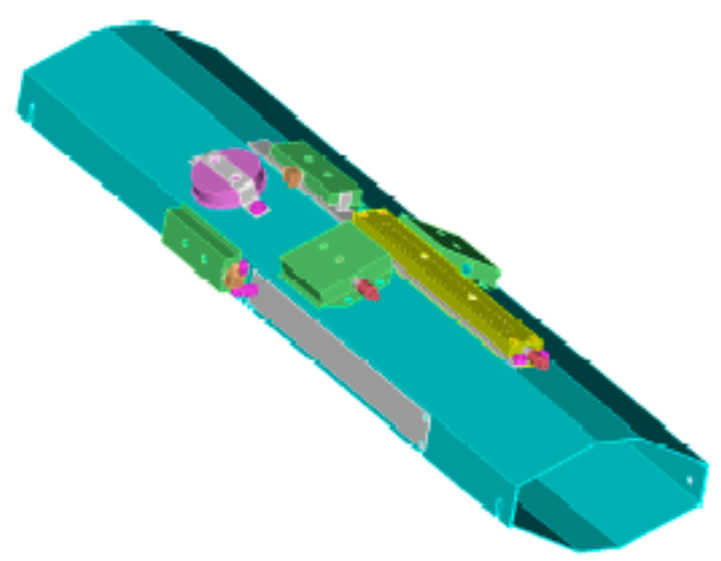

Fig. 3. 


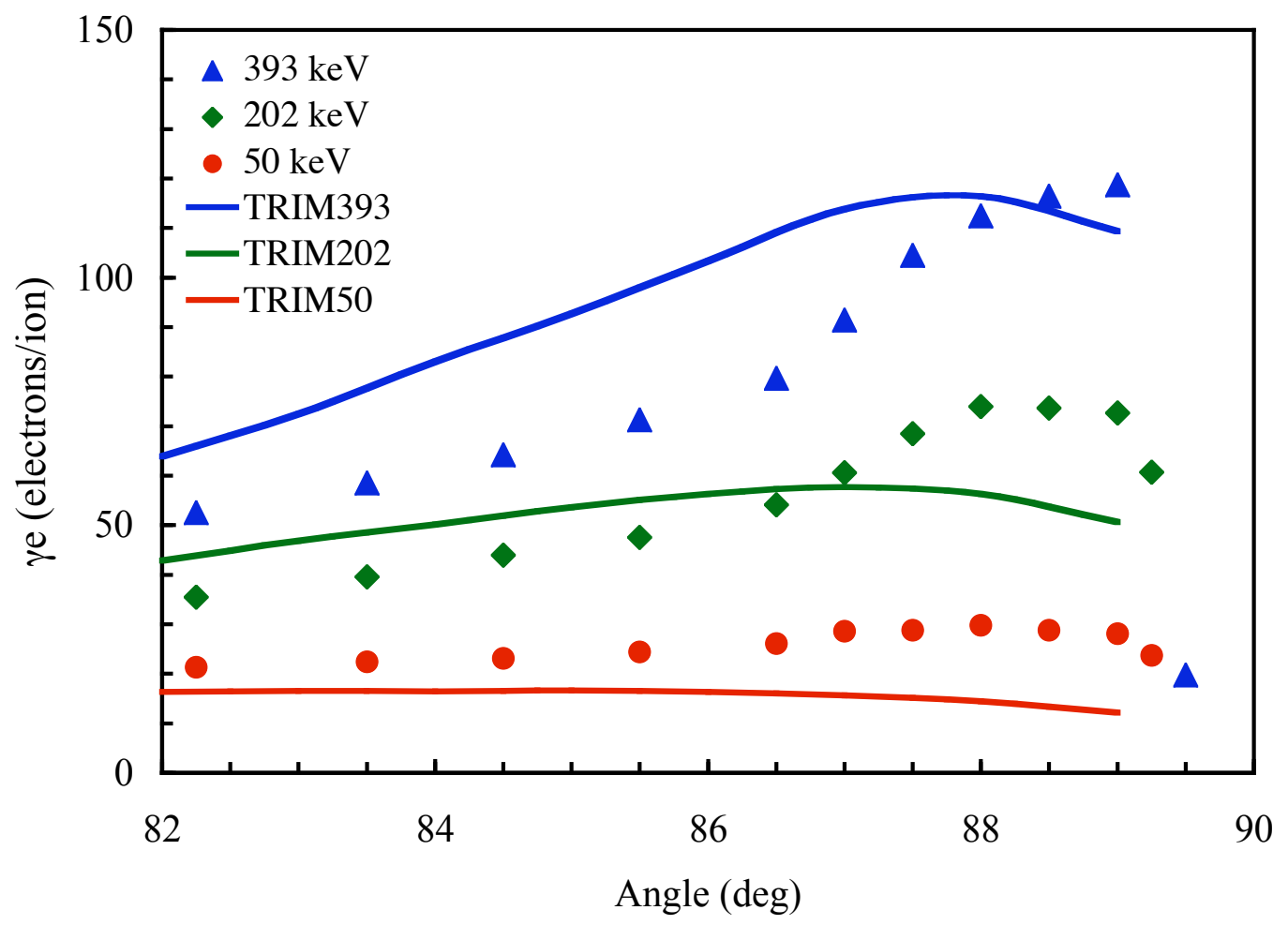

Fig. 4. 

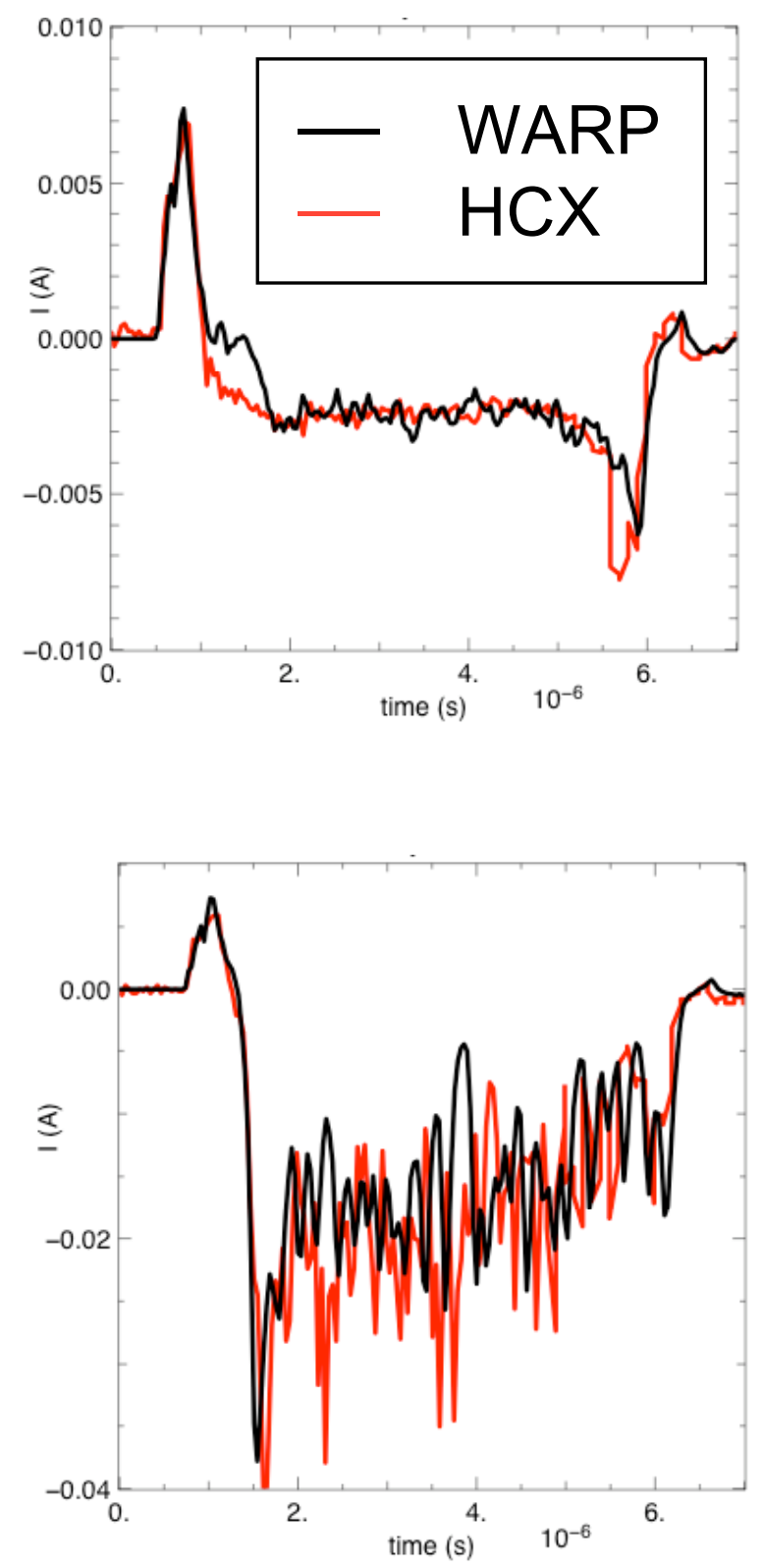

Fig. 5. 


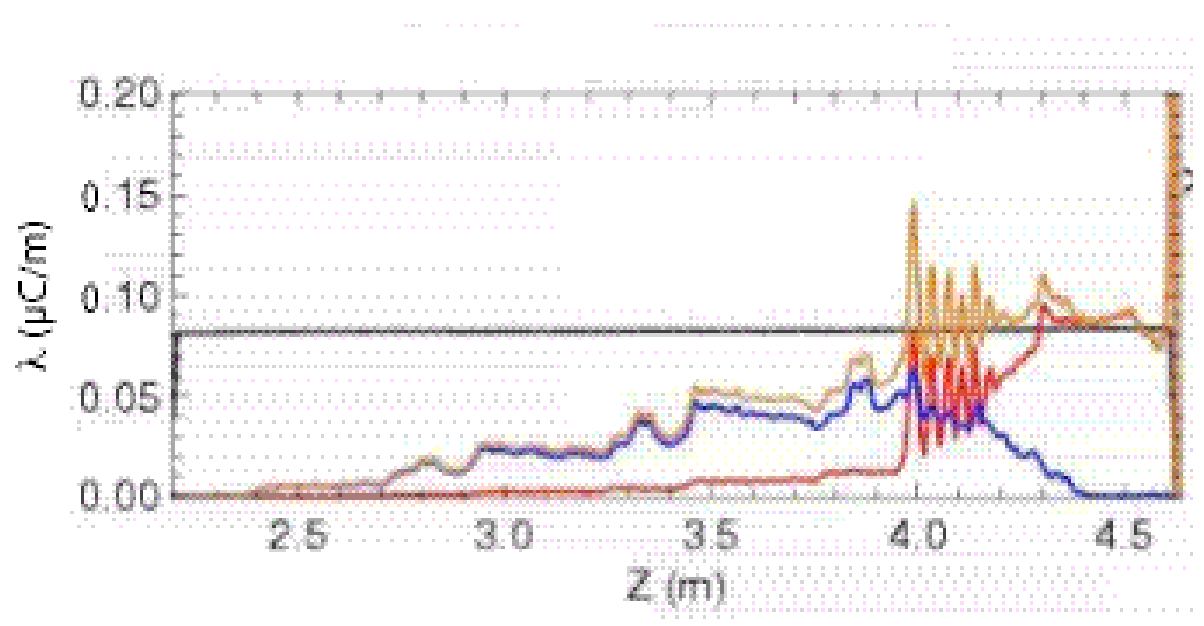

Fig. 6

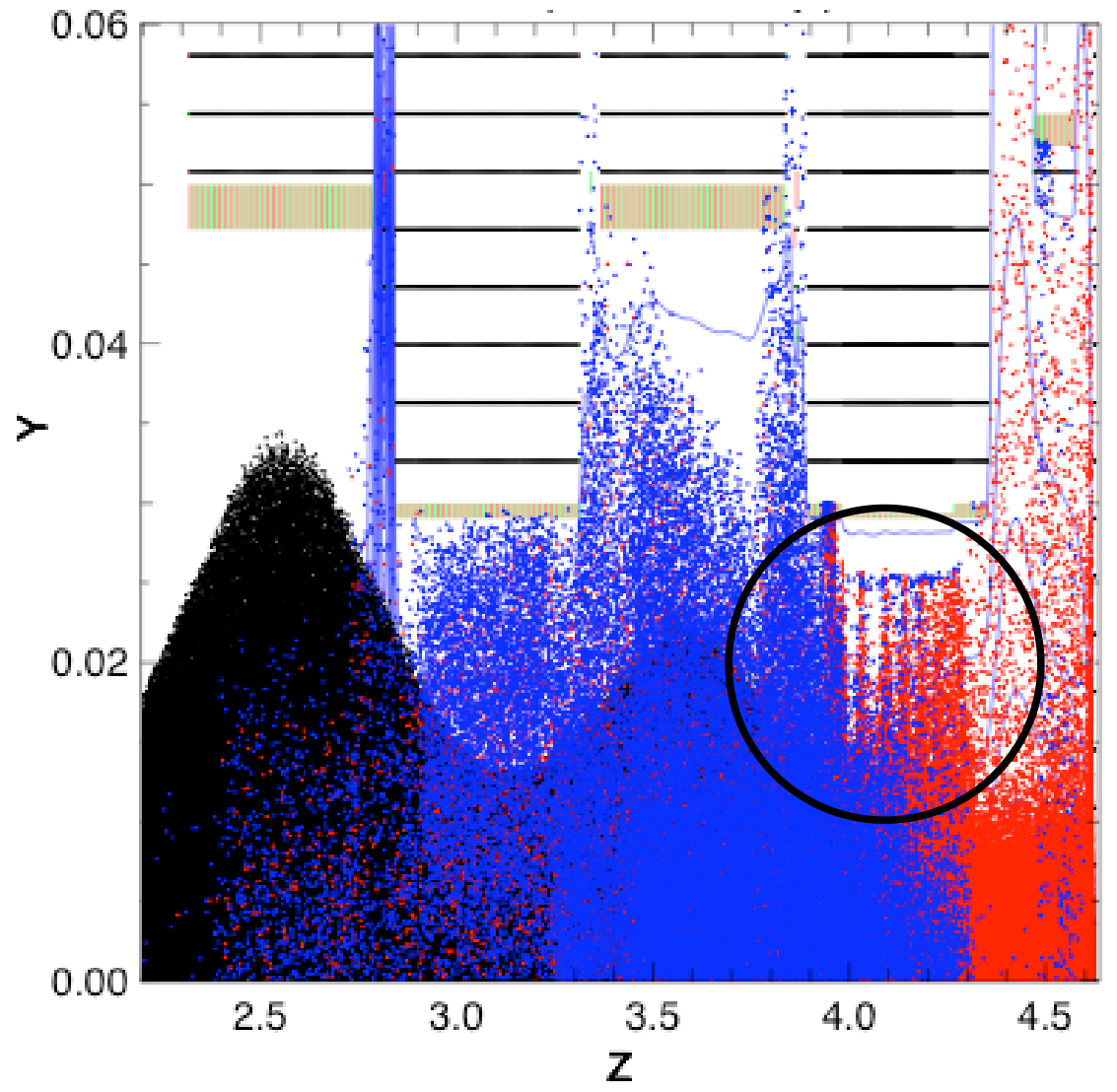

Fig. 7 


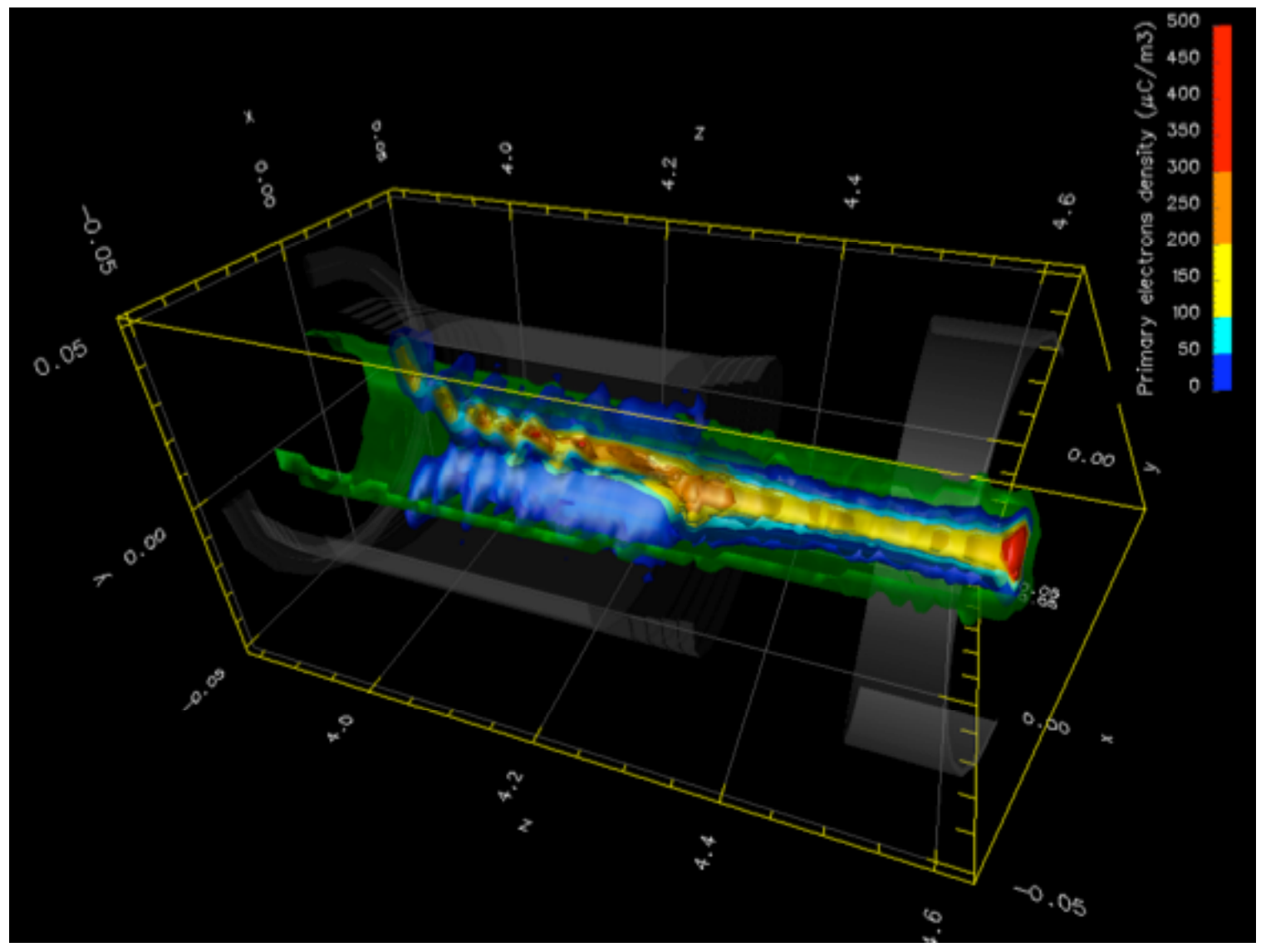

Fig. 8. 


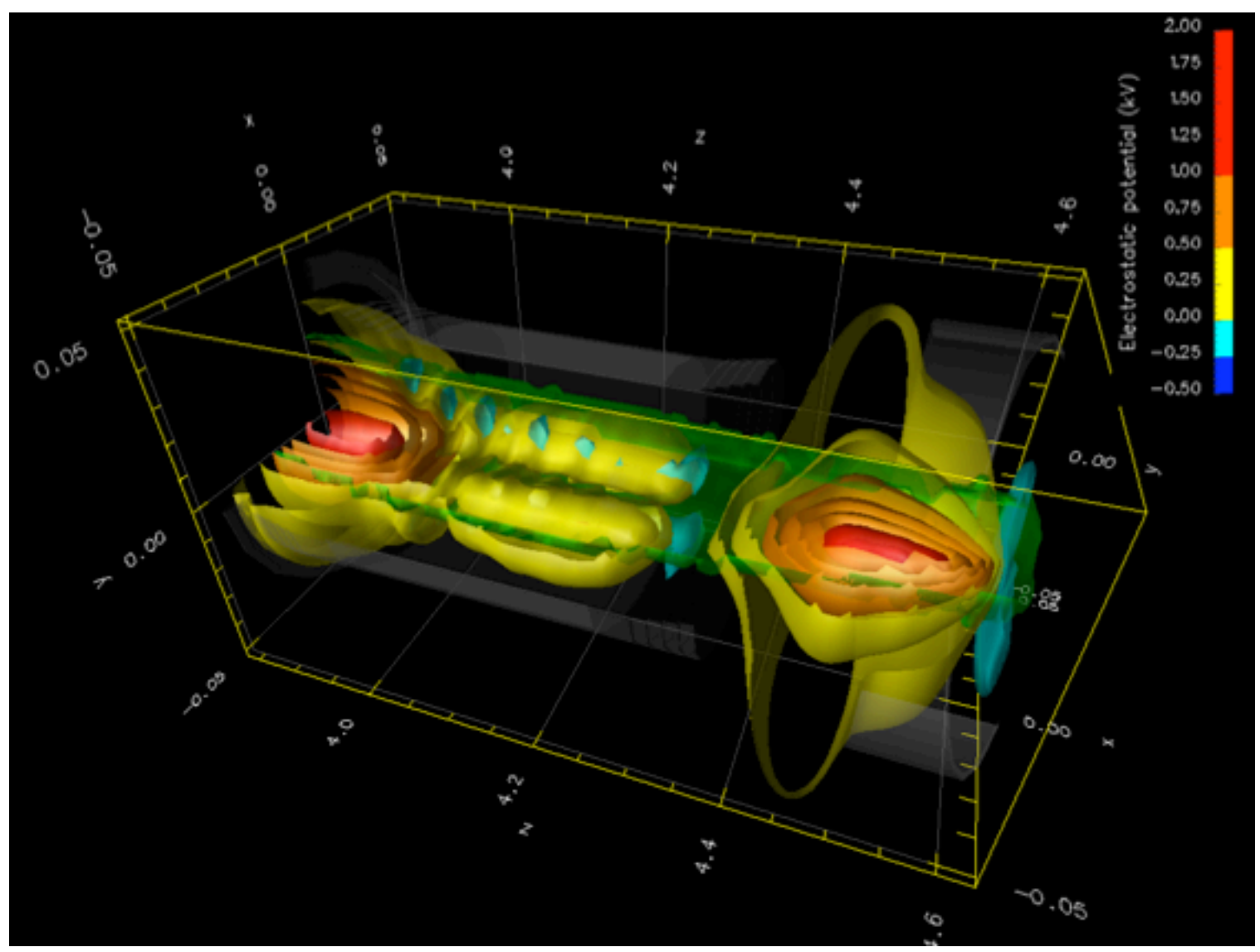

Fig. 9. 


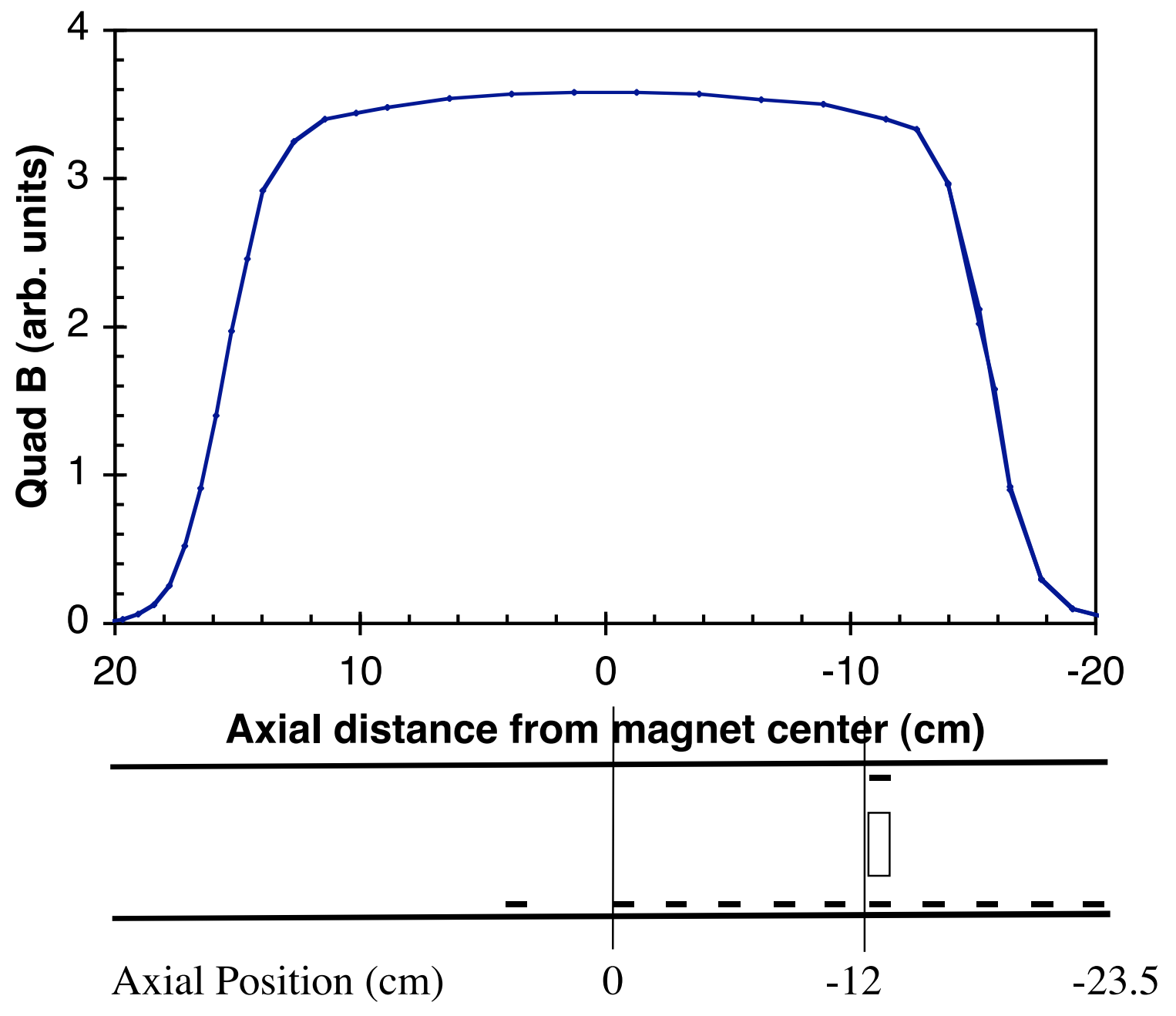

Fig. 10. 

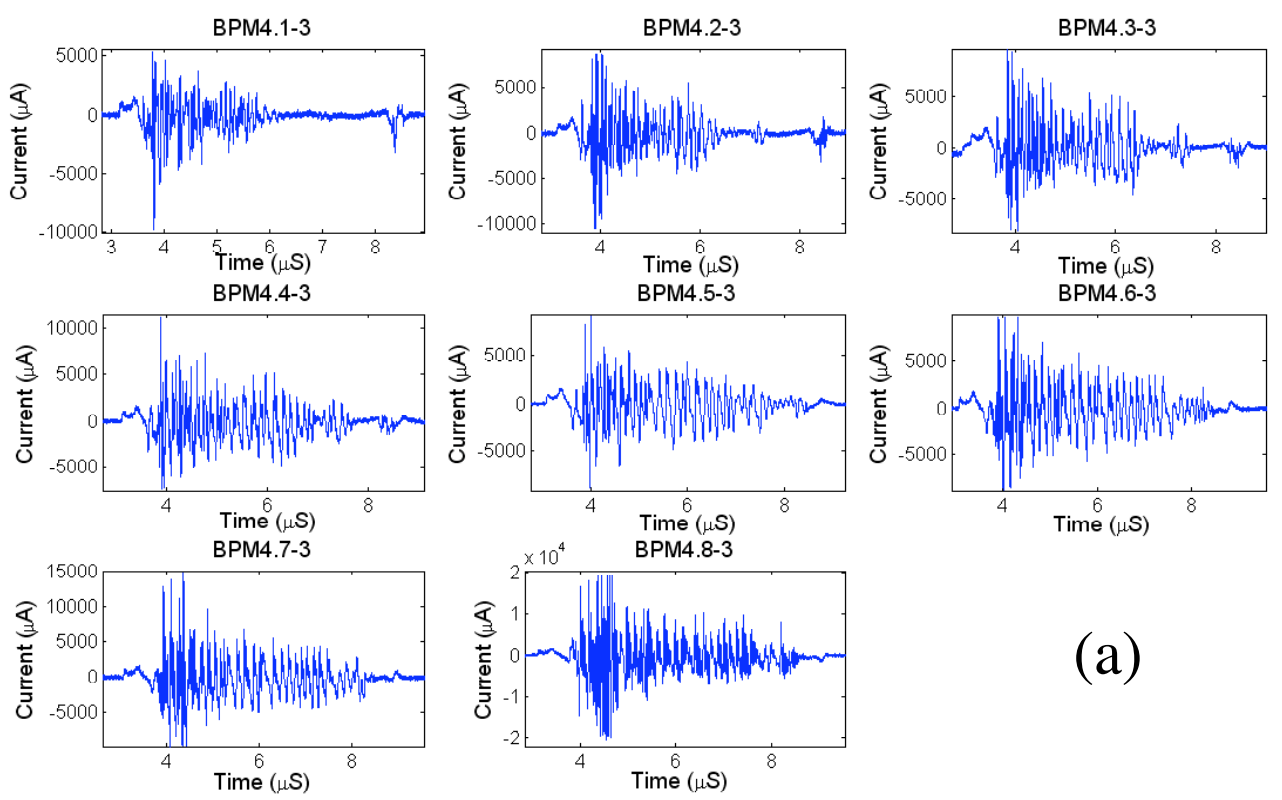

(a)
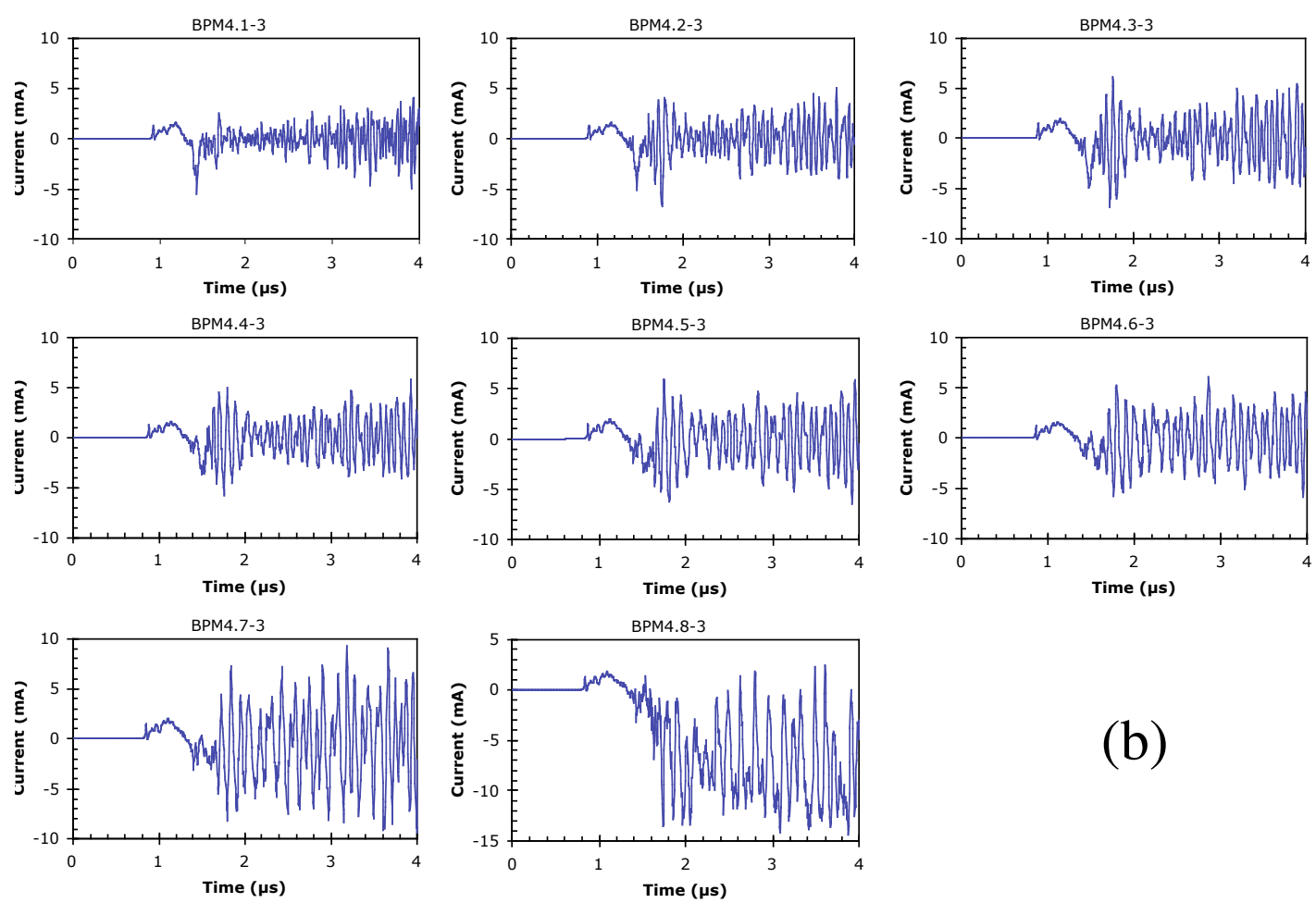

(b)

Fig. $11 \mathrm{a} \& \mathrm{~b}$ 


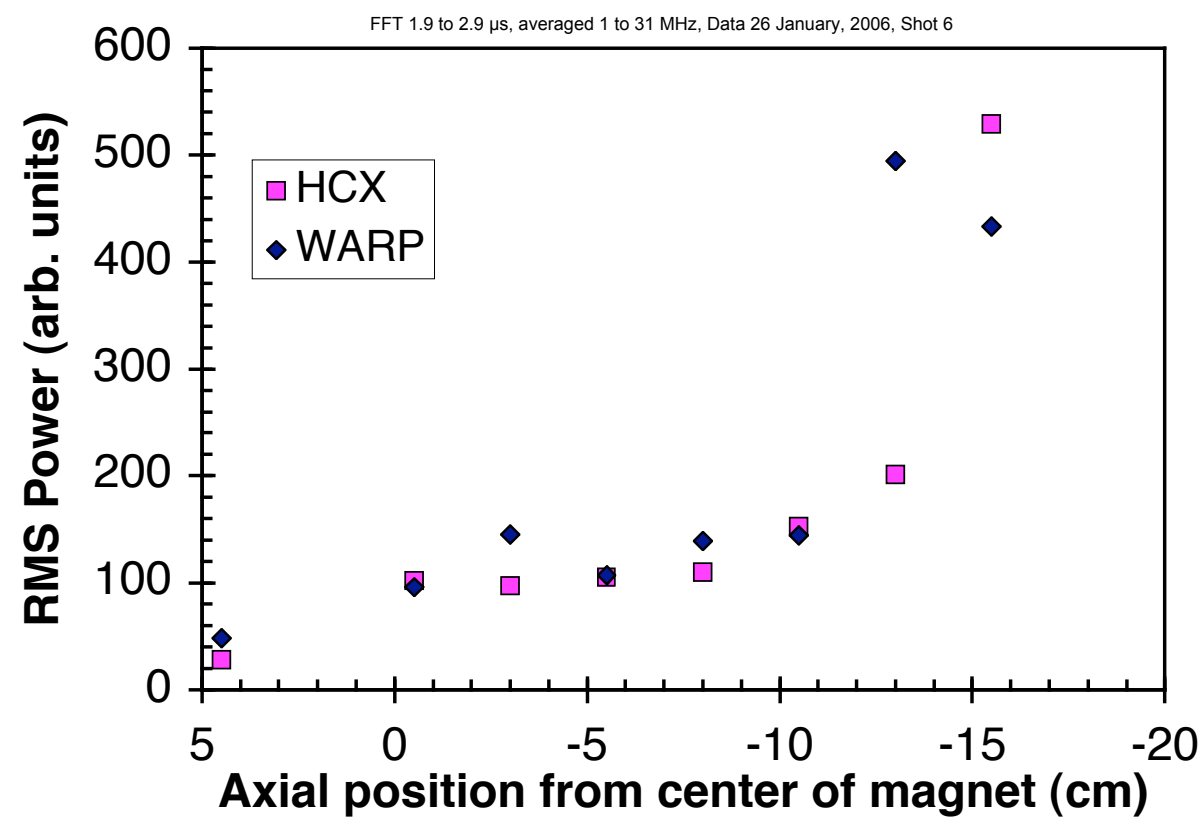

Fig. 12 


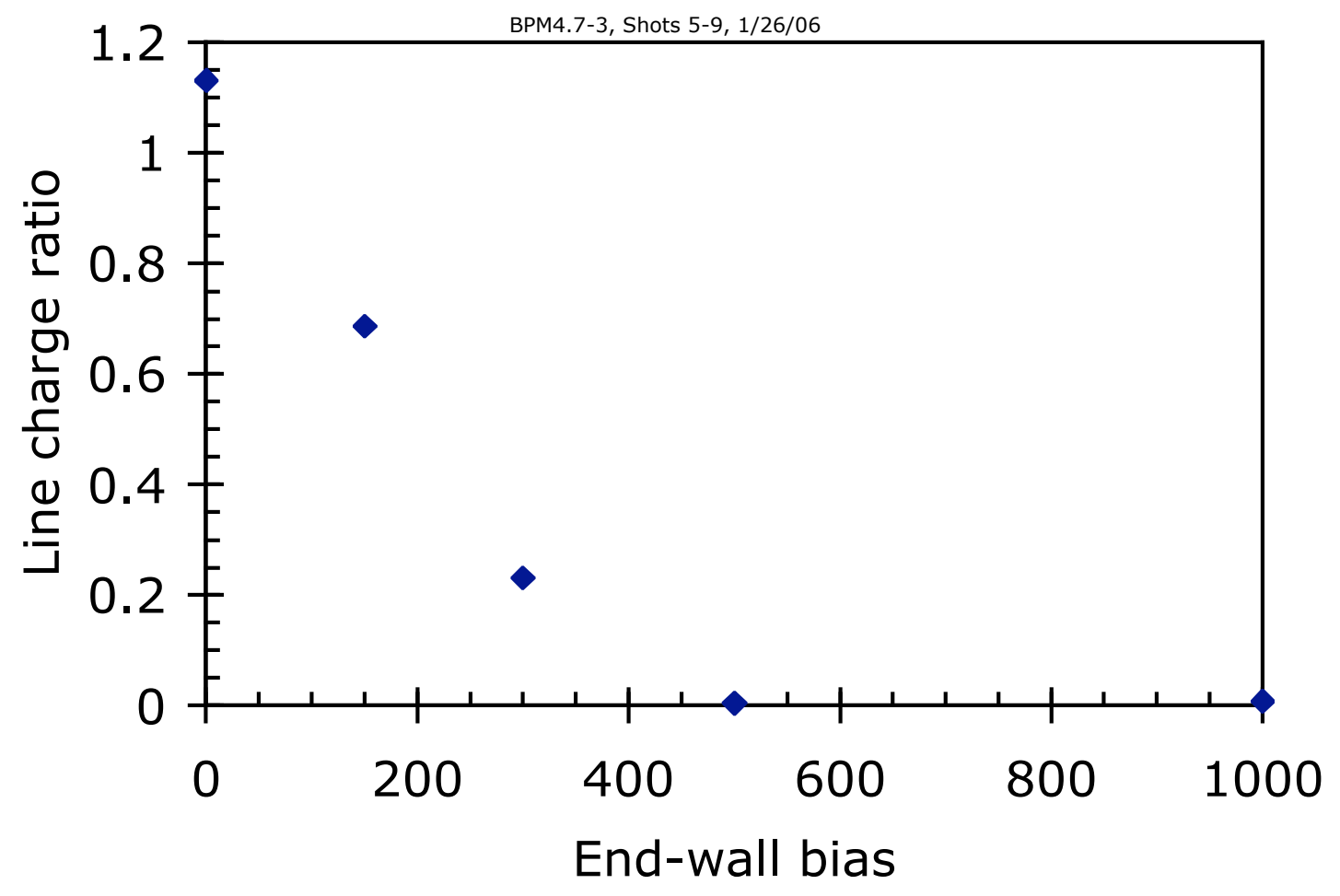

Fig. 13. 